\title{
Producción de azúcar de caña en El Salvador y sur relación con la variabilidad de la Actividad Solar y Geomagnética: Un enfoque de la Bioeconomía y el Cambio Climático
}

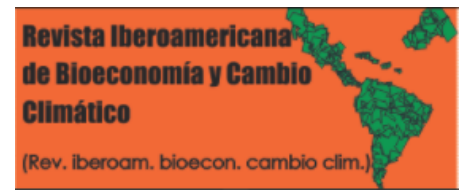

\author{
Cane sugar production in El Salvador and its relationship \\ with the variability of Solar and Geomagnetic Activity: An \\ approach to Bio economics and Climate Change
}

\author{
Sierra, Pablo; Marinero O, Edgar Antonio; Sol S, Ángel; Zuniga- \\ Gonzalez, Carlos A; Editor Académico Prof. Dr. Juan Manuel \\ Zaldivar-Cruz
}

\author{
Pablo Sierra \\ sierrafp@gmail.com \\ Instituto de Geofísica y Astronomía, AMA-CITMA, \\ Cuba, Cuba \\ Edgar Antonio Marinero O \\ edgar.marinero@ues.edu.sv \\ Universidad de El Salvador (CEIA)., El Salvador \\ Ángel Sol S \\ sol@colpos.mx \\ Colegio de Posgraduados, Tabasco, México, Mexico \\ Carlos A Zuniga-Gonzalez \\ czuniga@ev.unanleon.edu.ni \\ Universidad Nacional Autónoma de Nicaragua, León, \\ Nicaragua \\ Editor Académico Prof. Dr. Juan Manuel Zaldivar- \\ Cruz \\ Colegio de Postgraduados, Mexico
}

\author{
Revista Iberoamericana de Bioeconomía y Cambio \\ Climático \\ Universidad Nacional Autónoma de Nicaragua, León, Nicaragua \\ ISSN-e: 2410-7980 \\ Periodicidad: Semestral \\ vol. 5, núm. 10, 2019 \\ czuniga@ct.unanleon.edu.ni
}

Recepción: 15 Julio 2019

Aprobación: 04 Diciembre 2019

URL: http://portal.amelica.org/ameli/journal/394/3941757001/

DOI: https://doi.org/10.5377/ribcc.v5i10.8946

Autor de correspondencia: sierrafp@gmail.com
Resumen: El presente trabajo es una contribución de la Bioeconomía en el sendero productivo de Eco intensificación, donde los efectos de la variabilidad climática inciden en los niveles de productividad de la producción de Caña de Azúcar. La Actividad eruptiva del Sol y el Clima Espacial son importantes elementos del medio ambiente cósmico que inciden en la variabilidad del clima del planeta y numerosos procesos de la Biosfera y el Medio Ambiente. Se analizaron las series de tiempo de la productividad en la industria azucarera en El Salvador para un período de 31 años (1985-2015) a partir de los datos publicados en el Anuario de Estadística Agropecuaria, D.G.E.AM.A.G, en relación con el régimen lluvioso para ese mismo periodo y con dos índices del Clima Espacial. El trabajo se realizó en distintas fases en Cuba, El Salvador y México. El objetivo del trabajo, consistió en valorar la hipótesis que establece la asociación entre estas variables (series de producción azucarera e índices del clima espacial) y al mismo tiempo confirmar la posible influencia para este territorio centroamericano, y la muestra con que se contrasto. Se muestra que existe una estrecha sincronía entre la Actividad Solar y Geomagnética y los índices de producción de caña de azúcar, melaza y azúcar, manifestándose claramente en estas últimas un ciclo multianual de aproximadamente 10 a 12 años.

Palabras clave: Cambio Climático, Geomagnetismo, Heliobiología, Bioeconomia, Ecointensificaciòn.

Abstract: The present work is a contribution of the Bioeconomy in the productive path of Eco intensification, where the effects of climatic variability affect the productivity levels of sugarcane production. The eruptive Activity of the Sun and Space Weather are important elements of the cosmic environment that affect the variability of the planet's climate and numerous processes of the Biosphere and the Environment. The time series of productivity in the sugar industry in El Salvador for a period of 31 years (1985-2015) were analyzed from the data published in the Yearbook of Agricultural Statistics, DGEA-MAG, in relation to the rainy regime for that same period and with two indexes of the Space Weather. The work was carried out in 
different phases in Cuba, El Salvador and Mexico. The objective of the work was to assess the hypothesis that establishes the associations between these variables (sugar production series and spatial climate indices) and at the same time confirm the possible influence for this Central American territory, and the sample with which it was contrasted. It is shown that there is a close synchrony between the Solar and Geomagnetic Activity and the sugarcane, molasses and sugar production indices, clearly manifesting in these last ones a multi-year cycle of approximately 10 to 12 years.

Keywords: Climate Change, Geomagnetism, Heliobiology, Bioeconomy, Ecointensification.

\section{INTRODUCCIÓN}

El conocimiento de los factores que de una u otra manera inciden en los resultados productivos de cualquier actividad humana redundará sin dudas en el mejor aprovechamiento de los recursos utilizados en la misma, revirtiéndose en un mejor índice de costo-beneficio. En términos de la Bioeconomia la eco intensificación implica considerar factores de la biomasa para agregar valor a la producción.

La eco intensificación es referida a las prácticas agronómicas dirigidas a mejorar el rendimiento ambiental de las actividades agrícolas sin sacrificar los niveles de producción / productividad existente. Un indicador clave es la siembra directa, así podemos mencionar, por lo menos al 2006 a Brazil, Argentina, Paraguay, Bolivia, Venezuela, Chile y Colombia, según datos de la FAO evidencia esta afirmación (Dios, 2015). La agroindustria constituye una actividad productiva que depende mucho no sólo del empleo de tecnologías de cultivo, mantenimiento y buenas prácticas de cosecha y posterior procesamiento antes de llegar el producto final al mercado, así como de la utilización de las técnicas aplicadas en el control de irrigación, nutrientes, plagas y epifitas, sino también del Medio Ambiente en que se realiza dicha actividad en su conjunto, y cuando mencionamos Medio Ambiente, estamos incluyendo el electromagnético natural impuesto por las condiciones cíclicas del Clima Espacial, de la irradiación solar, de la actividad eruptiva del Sol y los procesos electromagnéticos propios del planeta, aspectos estos últimos generalmente ignorados en la literatura y en la vida práctica.

No son pocos los científicos, que en su afán de llevar a la práctica social los conocimientos acumulados y en continuo avance, insisten en poner a la luz pública y sobre todo de quienes manejan la economía y la industria agrícola, aquellos resultados que obstinadamente muestran los reales nexos entre el cosmos cercano (el Sol, la Luna y el Medio Ambiente Circunterrestre, entre otros elementos), su ciclicidad y pronóstico tal como lo expone (Vitinskii 1873; Vitinskii 1983, Chizhevskii, A. 1973, Chizhevskii, A. 1940,Piccardi, G. 1971,Virden L. Harrison. 1976) magistralmente en sus trabajos.

En ninguno de los trabajos consultados se concluye que dichas influencias físicas determinan aquel u otro fenómeno o actividad o incluso aspectos de la biosfera relacionados con la salud vegetal, animal o humana, sino que dicha actividad, de carácter cíclico o esporádico, imprime su comportamiento en numerosos procesos en el planeta, sean estos geofísicos, climáticos o biológicos, esto es, una influencia que se superpone o combina con los procesos endógenos de estos sistemas, trayendo como resultado determinados comportamientos "anómalos" difíciles de explicar por otras vías; así surgió, por cierto, el descubrimiento de que la actividad cíclica de aproximadamente 10 a 12 años del Sol modulaba, de alguna manera, el crecimiento

NotAS DE AUTOR

sierrafp@gmail.com 
de los anillos de los árboles seculares y el precio del trigo en el Reino Unido a principios del siglo IXX, cuando el astrónomo alemán William Hershel (1738-1822) observó que el precio del trigo en el mercado londinense marchaba sincrónicamente con el número de manchas que aparecían en el Sol año tras año. Tales descubrimiento dieron inicio a principios del pasado siglo a investigaciones por parte de insignes naturalistas tales como L.A. Chizhevski (1973), quien mostró certeramente cómo la actividad del Sol se inmiscuía en los procesos de aparición y desaparición de plagas y epidemias en Europa, en el comportamiento inexplicable del rendimiento de cosechas y otros muchos aspectos de la actividad humana, todo lo cual fue material excelente para presentar su Tesina de doctorado en el año 1915 en la Universidad de Moscú ("Investigaciones sobre la Periodicidad de los Procesos Globales Periódicos"). Su titánica labor dio por resultado la aceptación del término poco conocido de "Heliobiología", que se traduce como la Relación Sol - Biosfera, concepto éste último introducido por el también Naturalista ucraniano V. I. Verdadsky (1863-1945), quien puntualizó que “...en la Tierra no hay organismo vivo alguno que se encuentre en estado libre, sin estar vinculado indisolublemente con el medio material-energético. En nuestro siglo la biosfera se entiende de una manera completamente nueva. Se revela como fenómeno planetario de carácter cósmico" (Piccardi, G. 1971).

A estos descubrimientos iníciales, poco conocidos y comprendidos entonces, le siguieron, en las primeras décadas del pasado siglo, otros muchos aportes, realizados por científicos de los más diversos países y especialidades, a los que relacionarlos todos aquí sería muy extenso, pero sí debemos mencionar, entre otros al italiano Piccardi G. (1962) y S. Arrhenius (1859-1927), premio Nobel de Química, algunos de los cuales incursionaron en el comportamiento del clima del planeta en todas sus variables físicas, la Edafología, Agricultura, Rendimiento pesquero, modificación de las propiedades del agua, salud humana (patologías cardiovasculares, neurológicas, crecimiento y desarrollo, epidemias, entre otras muchas vertientes), así como resultados básicos bajo condiciones de modelación en el laboratorio.

De manera que el cúmulo de resultados confirmados por diferentes vías y autores han establecido una buena coherencia que permite en la actualidad afirmar que los nexos entre la Actividad Solar, el Clima y la Biosfera es una realidad que hay que tener en cuenta y llevar a la práctica los mismos por parte de los especialistas e instituciones encargadas de tomar decisiones en lo referente al clima, el Medio Ambiente, la economía y la salud humana, entre otros aspectos del quehacer humano.

En nuestra área geográfica han sido realizadas algunas investigaciones tanto en el aspecto de salud humana como en el económico, confirmándose de esta manera que en las bajas latitudes geográficas tropicales también se ven reflejados estos nexos en cuanto a la agroindustria Virden L. Harrison (1976). quien confirmó la hipótesis de la influencia que nos ocupa, con cuatro tipos de cosechas (algodón, arroz, trigo y maíz) en varios Estados de Estados Unidos, incluyendo Texas, para un período de más de 100 años, encontrando en general y con variados grados de influencia, mayor producción en etapas de alta actividad solar y viceversa, lo que concuerda con resultados contemporáneos reportados en las últimas décadas, Sierra et al. (1999), Muthanna A. et al. (2016).Zuniga, C. et al (2014), Pustil'nik, L. \& Din, G. (2004).

En el presente reporte hemos abordado la asociación entre la actividad cíclica del Sol, el geomagnetismo terrestre y la producción de caña de azúcar en El Salvador durante un período de 31 años, para cuyo país y período hubimos de investigar previamente el régimen de lluvias con una muestra de 8 estaciones de registro también en relación con las mismas variables físicas del Clima Espacial (Sanchez, 2017) encontrando una asociación positiva que nos condujo a explorar algunas variables de la agroindustria en el mismo territorio, en busca de posible nexo causal al ser la lluvia un elemento esencial en la gestión agrícola.

La importancia práctica de los resultados obtenidos en este tipo de investigación son evidentes, representando una herramienta útil de pronóstico para la planificación de los recursos y del esfuerzo productivo ( $\mathrm{y}$ como información de utilidad en el enfrentamiento de las consecuencias del calentamiento global que nos afecta a todos), el cual se hace más eficiente si se acentúa durante los períodos en que se conoce, por pronóstico de la Actividad Solar, que la producción se acrecentará debido al factor externo del Clima Espacial, independientemente de otros factores endógenos. 


\section{Materiales y MÉtodo}

La base de datos original de la producción cañera para 31 años (1985 - 2015, ambos incluidos) y sus derivados para el territorio de El Salvador fue obtenida del Anuario de Estadística Agropecuaria, D.G.E.AM.A.G de dicho país. A partir de la misma se confeccionó un libro EXCEL con los datos originales (Área física de plantación, Estimado del volumen de caña obtenido, Rendimiento cañero, Producción de Azúcar, Producción de Melaza) en sus respectivas unidades de medida originales.

Por tratarse de cifras con muchos dígitos y por no ser de interés para la presente exploración los valores absolutos de las variables, sino su variabilidad porcentual multianual, optamos por normalizarlas todas, creando una página Excel con los datos ya normalizados y a partir de las mismas se calculó dos nuevas variables no contenidas en la data original: Rendimiento de Azúcar y Rendimiento de Melaza. Vista la destacada tendencia de las variables se optó por suprimir la misma de manera de poder evidenciar mejor tanto gráficamente como en los resultados estadísticos la variabilidad multianual presente en ellas, todo lo cual se indica en los gráficos y resultados estadísticos correspondientes.

Como variables independientes, representativas de la Actividad Solar (AS) y su variabilidad, se tomó el Promedio Anual del Número de Woolf(W) que caracteriza el número y área de las manchas en el disco visible del Sol, el Promedio Anual del Flujo de Radioemisión Solar (F) en la longitud de onda de 10Cm. y se utilizó también como variable representativa de la Actividad Geomagnética Planetaria (AGP), que es consecuencia de la efectividad de la AS en el planeta, al índice Promedio Anual de "Ap".

Para las tres variables se obtuvo los promedios anuales a partir de los datos diarios o trihorarios en el caso de Ap, siendo las fuentes de los mismos http://www.sidc.be/silso/datafiles; http:// www.geomag.bgs.ac.uk/.html respectivamente.

\section{Resultados}

Todos los valores aparecen normalizados a los registros máximos del período. La línea segmentada representa el ajuste lineal de la tendencia para cada caso. 


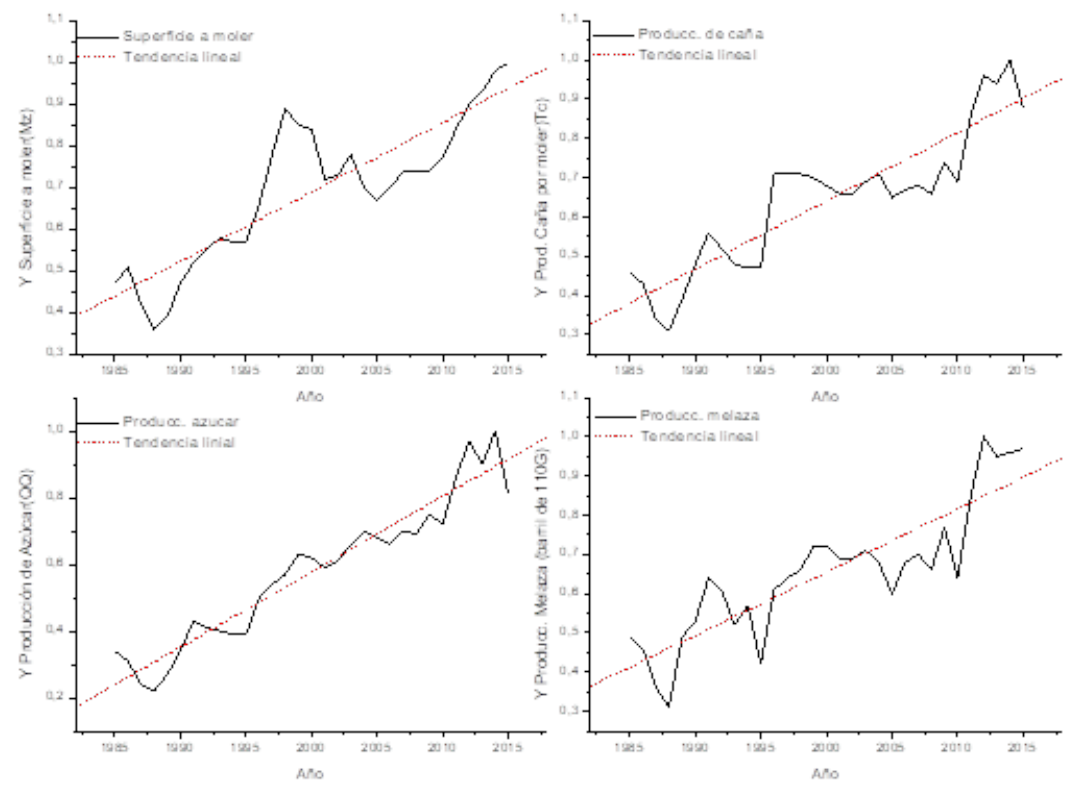

FIG. 1

Variables de producción y de área cultivada reportada

Todos los valores aparecen normalizados a los registros máximos del período. La línea segmentada representa el ajuste lineal de la tendencia para cada caso. Fig 2
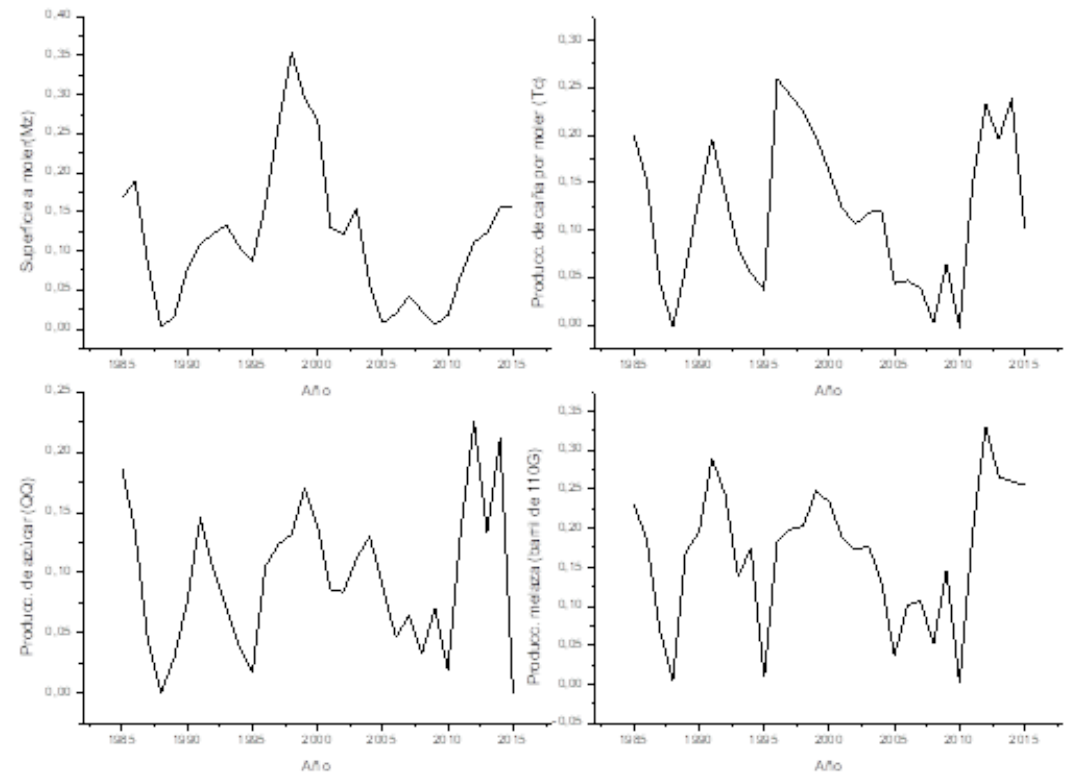

FIG. 2

Variables de producción y de área cultivada reportada.

Todos los valores aparecen normalizados a los registros máximos del período, en este caso suprimida la tendencia que se observa en la Fig. 1, lo que permite destacar la variabilidad porcentual en todos los casos.Fig 3 


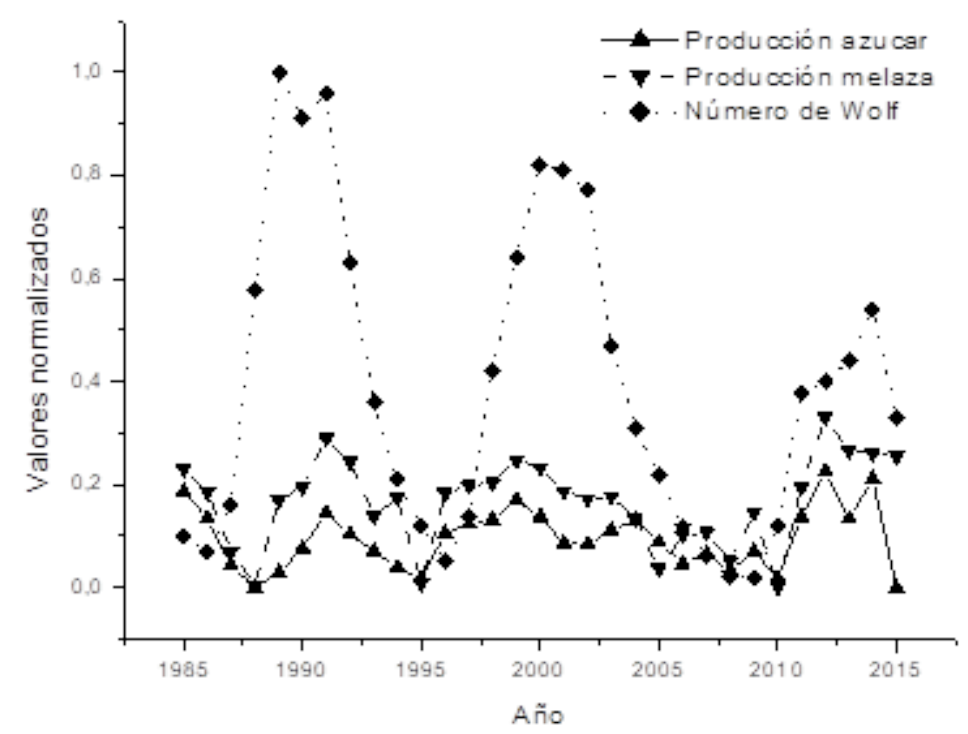

FIG. 3

Superposición gráfica de las variables de Producción y de Actividad Solar para todo el período

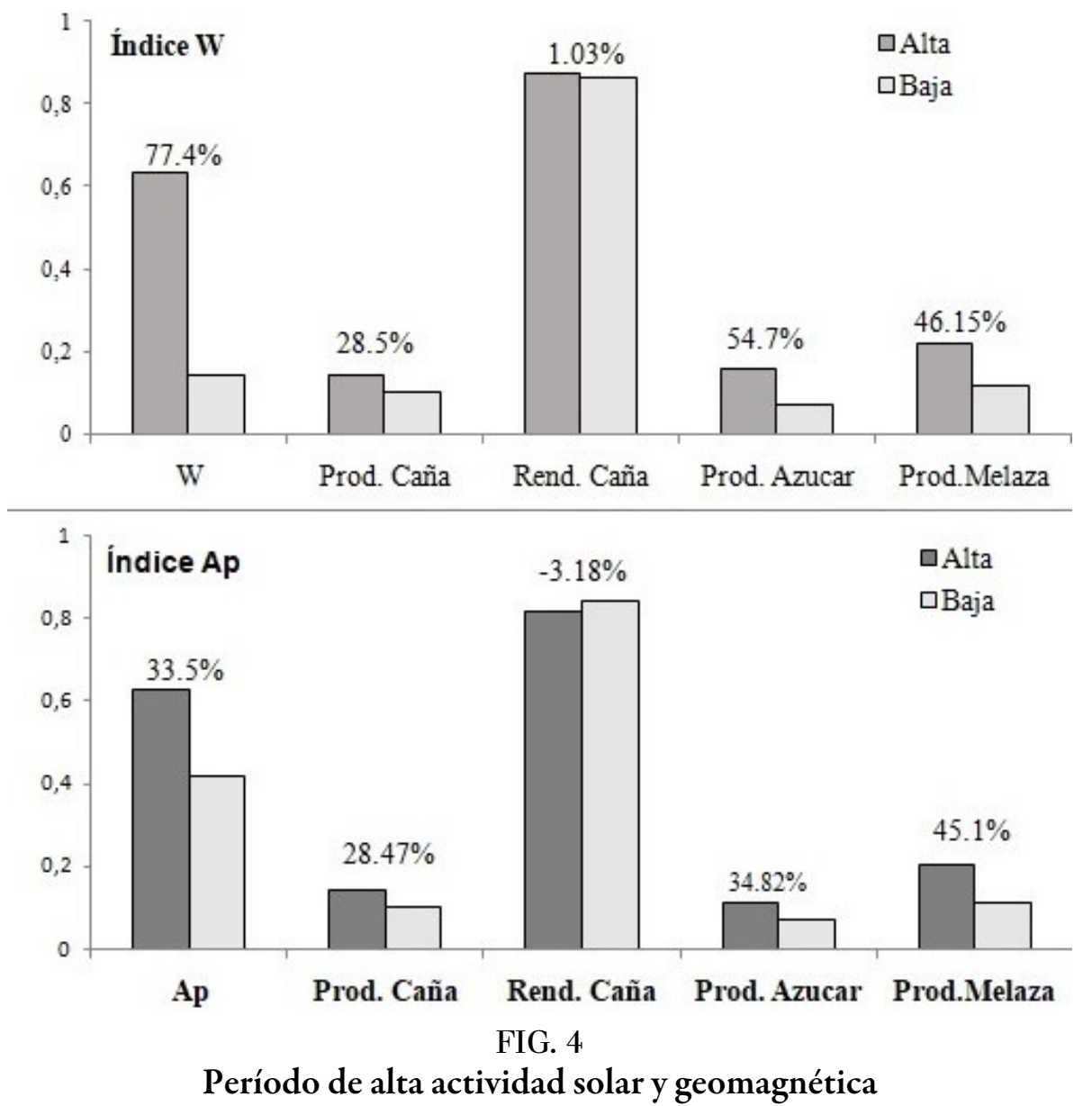

En la figura 4 se observa los resultado de superponer los períodos de alta actividad solar y geomagnética por separado y los correspondientes de producción y productividad de caña, en comparación con los períodos 
de baja actividad. Todas las magnitudes aparecen normalizadas. Los valores porcentuales sobre las barras representan las diferencias entre los períodos en comparación.Tabla 1

TABLA 1

Resultado de los períodos obtenidos mediante Transformada de Fourier (FFT) para todas las variables implicadas en el trabajo.

\begin{tabular}{|c|c|c|c|c|c|c|}
\hline VARIABLES & PERÍO & $D O(A$ & NOS) & & & \\
\hline Superficie a moler & 16 & 8 & 5.3 & 4 & 2.9 & 2.5 \\
\hline Caña por moler & 16 & 8 & 5.3 & 3.6 & & 2.5 \\
\hline $\begin{array}{l}\text { Rendimiento de } \\
\text { caña }\end{array}$ & - & - & - & - & - & - \\
\hline $\begin{array}{l}\text { Producción de } \\
\text { azúcar }\end{array}$ & 12.8 & 7.11 & & & & 2.6 \\
\hline $\begin{array}{l}\text { Producción de } \\
\text { melaza }\end{array}$ & 12.8 & 7.11 & & & 3.05 & \\
\hline $\begin{array}{l}\text { Productividad de } \\
\text { azúcar }\end{array}$ & - & - & - & - & - & - \\
\hline Índice solar $W$ & 12.8 & 7.11 & & 4 & & \\
\hline $\begin{array}{l}\text { Índice solar Flujo } \\
\text { Radio }\end{array}$ & 12.8 & 7.11 & & & & \\
\hline $\begin{array}{l}\text { Índice } \\
\text { geomagnético Ap }\end{array}$ & 10.6 & & 5.33 & 4 & 2.9 & 2.4 \\
\hline
\end{tabular}
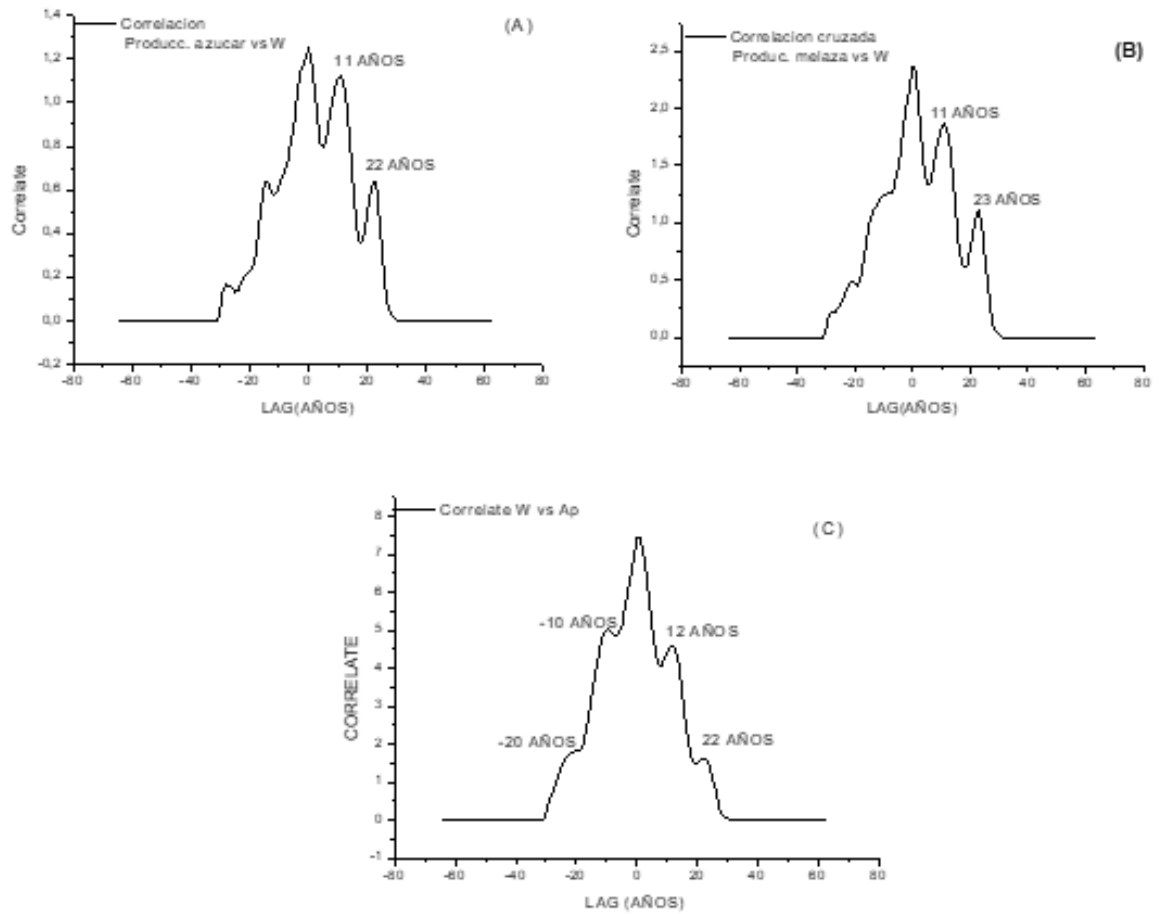

FIG. 5

Correlación cruzada entre el índice solar $\mathrm{W}$ y la producción de azúcar A y melaza B para el periodo analizado.

En la figura 5 se observan los resultado de la correlación cruzada entre el índice solar W y la producción de azúcar (A) y de melaza (B) para el período analizado. La asimetría observada se debe a la diferencia de duración de los ciclos de Actividad Solar y Geomagnética presentes para este período, lo cual es posible corroborar en 
el gráfico $\mathrm{C}$, donde, a modo de control, se muestra la correlación cruzada entre las variables independientes W y Ap. (Microcal Origin 6).

\section{Conclusiones}

Los resultados mostrados confirman la hipótesis de asociación causal entre la variabilidad multianual de la Actividad Solar y Geomagnética y la producción de caña, azúcar y melaza para la muestra utilizada, independientemente de la tendencia de estas últimas y a la vez se evidencia que los ritmos quinquenales encontrados en el régimen de lluvias en El Salvador (Sánchez et al. 2017) para los 31 años estudiados concuerdan con los ritmos que presenta la producción cañera, los cuales están también presentes en el Clima Espacial.

Consideramos que dada la diferencia encontrada en la producción para uno y otro período de perturbación en el Clima Espacial, del orden hasta del 20\%, es válido y conveniente explorar este aspecto para otras producciones agrícolas y a la vez someter a un pilotaje experimental en la toma de decisiones en cuanto a la planificación del esfuerzo productivo para plazos comprendidos en períodos de alta y baja actividad solar, lo cual permitiría no sólo confirmar lo obtenido en este trabajo, sino también explotar el conocimiento de la asociación encontrada y su repercusión económica. Todo ello contribuirá en cierta medida a la mitigación del aspecto negativo que imprime el Cambio Climático en la producción de alimentos.

En términos de la Bioeconomía, este estudio representa un nuevo enfoque de valorar la producción en el sendero de la Eco intensificación donde la actividad solar y geomagnética inciden en la producción de manera directa como una variable climática.

\section{ReFERENCIAS}

Chizhevskii, A. 1940. Cosmobiologie et Rythme du Milieu extérieur. Verhandlungen, Zweiten Konferenz der Internationalen Gesellschaft für Biologische Rhythmusforschung, am 25. und 26. August 1939, Utrecht, Holland, HolmgrenHj, editor. Acta med scand 1940; 108 (Suppl): 211-226.

Chizhevskii, A. 1973. El eco terrestre de las tormentas solares. Ed. "Misl" Moscú, 1973.

Muthanna, A. Al-Tameemi; Chukin V. 2016. Global wather cycle and solar activity variations. Journal of Atmospheric and Solar-Terrestrial Physics142 (2016) 55-59.

Piccardi, G. 1971. Actividad Solar y los test químicos. Influencia de la Actividad Solar en la Atmósfera y la Biosfera de la Tierra. Moscú, 1971, p. $141-147$.

Piccardi, G. 1962. The chemical basis of medical climatology. Springfield (Ill. USA, Charles C. Thomas..

Pustil'nik, L.A. \& Din, G.Y. (2004). Influence of solar activity on the state of the wheat market in medieval England. Sol Phys (2004) 223: 335.

Dios P. R., Alcaide, J. D., Jurado, M., Guijarro, A. P., Martinez-Paz, J., \& Zúniga-González, C. (2015). Aspectos medioambientales en los análisis de eficiencia. Revista Iberoamericana de Bioeconomía y Cambio Climàtico, $1(1)$.

Sierra, P., Sierra, S., Rodríguez R., Pérez P. 1999. Impacto Medioambiental de las Perturbaciones Heliogeofísicas. Consideraciones a partir de Resultados Observacionales. México, D.F. Rev. Geofísica, No. 50, Enero-Junio 1999, pp. 9-23..

Sanchez, Á. S., Sierra-Figueredo, P., \& Marinero-Orantes, E. A. (2017). Actividad solar y su asociación con el régimen de lluvias en El Salvador. Revista Iberoamericana de Bioeconomia y Cambio Climático, 3(6), 782-799.

Virden L. Harrison. 1976. Do sunspot cycles affect crop yields? Economic Research Service, U.S. Department of Agriculture. Agricultural Economic Report No. 327.

Vitinskii Yu. 1973. Ciclicidad y pronóstico de la Actividad Solar (ruso). Ed. "NAUKA". Leningrado 1973. 254p 
Pablo Sierra, et al. Producción de azúcar de caña en El Salvador y su relación con la variabilidad...

Vitinskii Yu. 1983. Actividad Solar y Biosfera (ruso). Actividad Solar. Moscú. Ed."Nauka", 1983. pp.179-192

Zúniga-González, C. A., Durán Zarabozo, O., Dios Palomares, R., Sol Sánchez, A., Guzman Moreno, M. A., Quiros, O., \& Montoya Gaviria, G. D. J. (2014). Estado del arte de la bioeconomía y el cambio climático (No. 1133-2016-92457, pp. 20-329). 